\title{
Erratum
}

\section{Erratum to "Preventive Strategies against Bleeding due to Nonvitamin K Antagonist Oral Anticoagulants"}

\author{
Sarah Lessire, ${ }^{1,2}$ Anne-Sophie Dincq, ${ }^{1}$ Jonathan Douxfils, ${ }^{2}$ Bérangère Devalet, ${ }^{2,3}$ \\ Jean-Baptiste Nicolas, ${ }^{4}$ Anne Spinewine, ${ }^{5,6}$ Anne-Sophie Larock, \\ Jean-Michel Dogné, ${ }^{2}$ Maximilien Gourdin, ${ }^{1}$ and François Mullier ${ }^{2,5,7}$ \\ ${ }^{1}$ Department of Anesthesiology, CHU Dinant-Godinne UCL Namur, Namur Thrombosis and Hemostasis Center (NTHC), \\ Namur Research Institute of Life Sciences (NARILIS), 5530 Yvoir, Belgium \\ ${ }^{2}$ Department of Pharmacy, Namur Thrombosis and Hemostasis Center (NTHC), Namur Research Institute of Life Sciences (NARILIS), \\ University of Namur, 5000 Namur, Belgium \\ ${ }^{3}$ Department of Hematology, CHU Dinant-Godinne UCL Namur, Namur Thrombosis and Hemostasis Center (NTHC), \\ Namur Research Institute of Life Sciences (NARILIS), 5530 Yvoir, Belgium \\ ${ }^{4}$ Department of Internal Medicine, CHU Dinant-Godinne UCL Namur, Namur Thrombosis and Hemostasis Center (NTHC), \\ Namur Research Institute of Life Sciences (NARILIS), 5530 Yvoir, Belgium \\ ${ }^{5}$ Department of Pharmacy, CHU Dinant-Godinne UCL Namur, Namur Thrombosis and Hemostasis Center (NTHC), \\ Namur Research Institute of Life Sciences (NARILIS), 5530 Yvoir, Belgium \\ ${ }^{6}$ Clinical Pharmacy Research Group, Louvain Drug Research Institute, Université Catholique de Louvain, 1200 Brussels, Belgium \\ ${ }^{7}$ Hematology Laboratory, CHU Dinant-Godinne UCL Namur, Namur Thrombosis and Hemostasis Center (NTHC), \\ Namur Research Institute of Life Sciences (NARILIS), 5530 Yvoir, Belgium
}

Correspondence should be addressed to François Mullier; mullierfrancois@gmail.com

Received 6 August 2014; Accepted 28 August 2014; Published 11 September 2014

Copyright (c) 2014 Sarah Lessire et al. This is an open access article distributed under the Creative Commons Attribution License, which permits unrestricted use, distribution, and reproduction in any medium, provided the original work is properly cited.

In the paper titled "preventive strategies against bleeding due to nonvitamin K antagonist oral anticoagulants," the authors' given first and last names were inverted. Here we provide the right authors' information as shown above. 


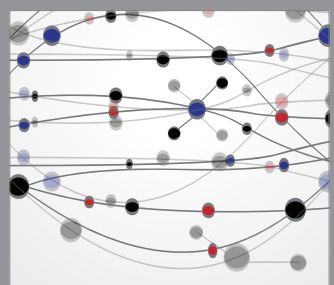

The Scientific World Journal
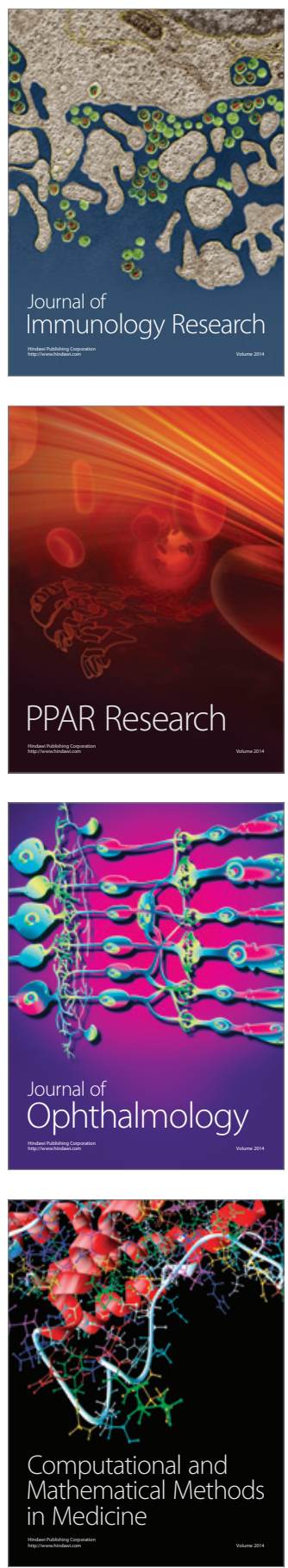

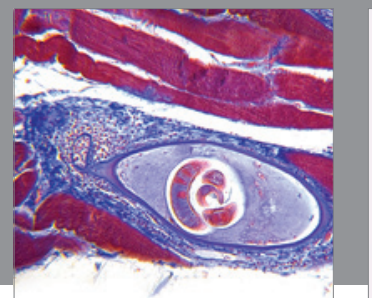

Gastroenterology

Research and Practice
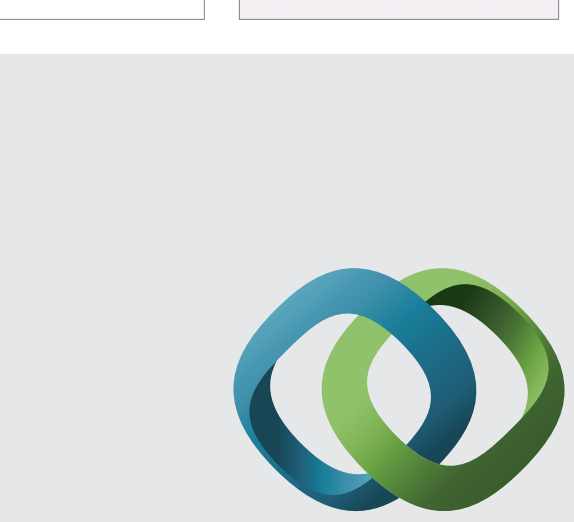

\section{Hindawi}

Submit your manuscripts at

http://www.hindawi.com
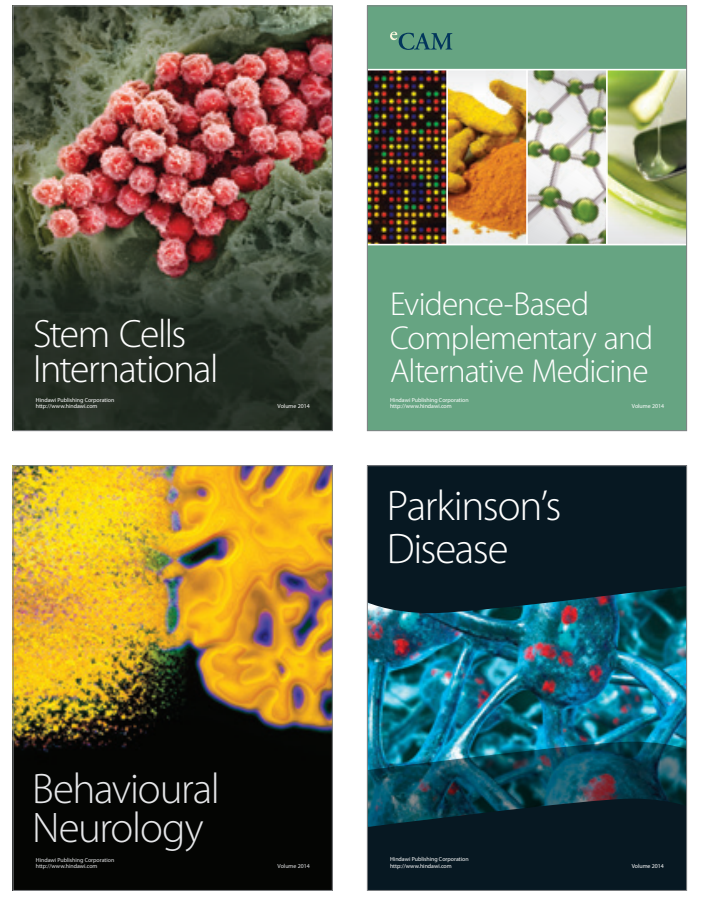
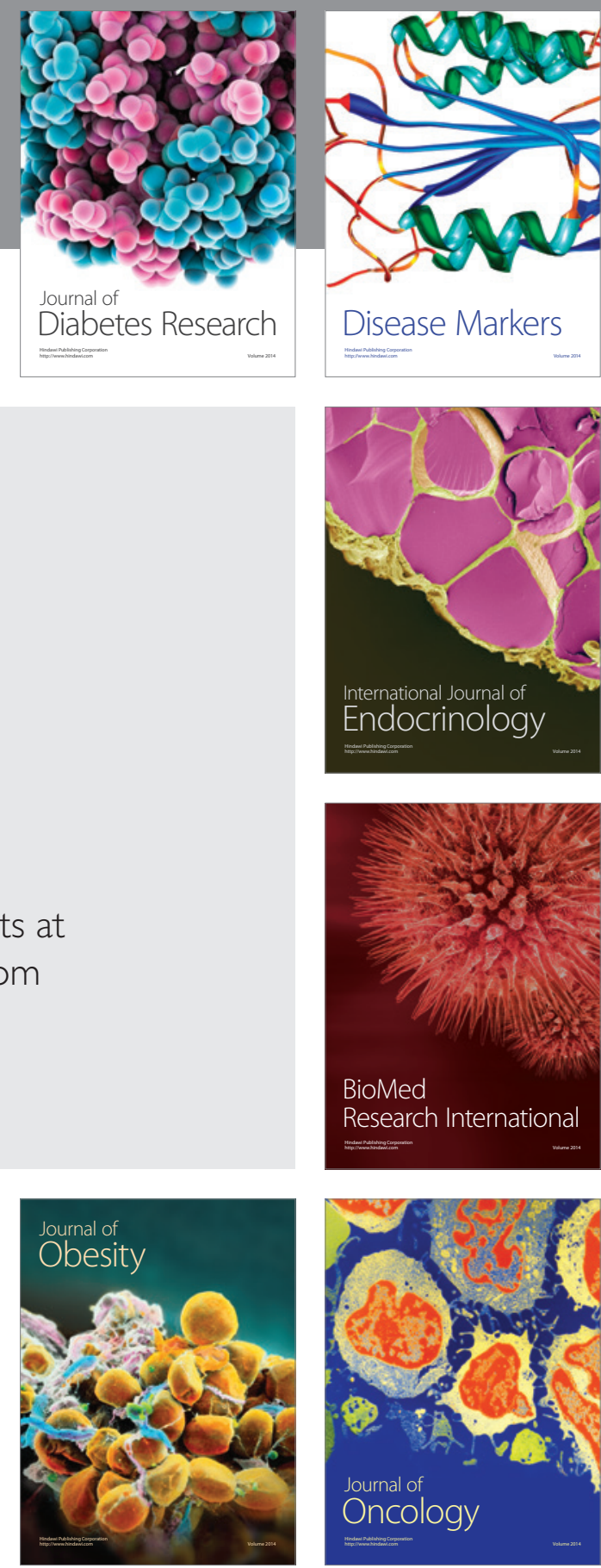

Disease Markers
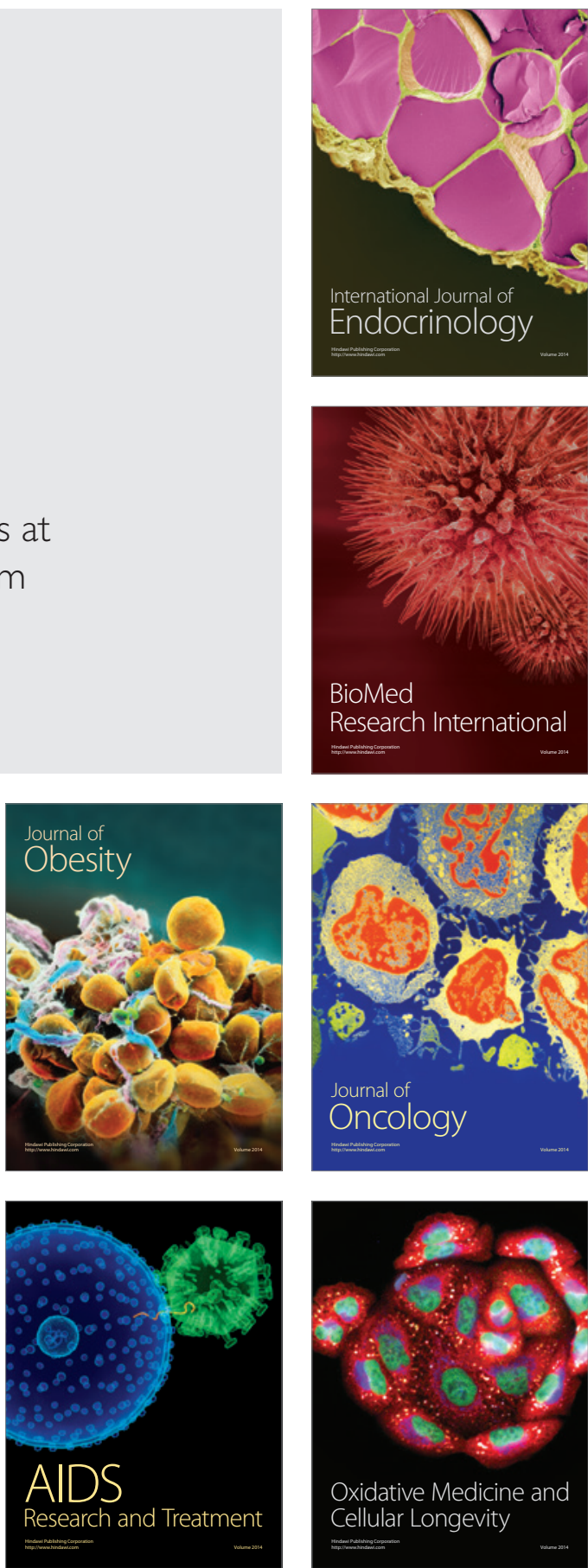